\title{
Controversial Features of Granular Superconductors Studied Through the Magnetic Properties of 2D-Josephson Junction Arrays
}

\author{
W. Maluf and F. M. Araujo-Moreira \\ Department of Physics, \\ Multidisciplinary Center for Development of Ceramic Materials-MCDCM \\ Universidade Federal de São Carlos \\ Caixa Postal 676, São Carlos SP, 13565-905, Brazil
}

Received on 28 February, 2002

\begin{abstract}
We have shown that the Paramagnetic Meissner Effect (PME) is directly associated with pinning, and not necessarily related to the presence of $\pi$-junctions. Through the study of the magnetic properties of two-dimensional Josephson junction arrays (2D-JJA) in the present work we show that, among the systems exhibiting PME, only those with sufficiently low dissipation and high capacitance will show dynamics reentrance. The concept of a critical state and its use in the interpretation of $\mathrm{AC}$ magnetization data in terms of a critical current density were introduced to derive the magnetic properties of hard type-II superconductors. In the critical state model proposed by Bean, flux lines penetrate into the sample and, due to the presence of disorder they give rise to a steady flux gradient. Here we show that in 2D-JJA this typical picture is valid only in short-range distances. For long-range distances, the picture of uniform flux fronts, as described by a critical state model, breaks down and the penetration of the magnetic field takes place through the growth of magnetic dendrites. De Gennes originally compared the slope of a pile of vortices to a sandpile, with the slope being proportional to the local magnitude of the critical current. Dynamical properties of the sand-pile problem have attracted new attention since it consists of a marginally stable system displaying self-organized criticality (SOC). In this case, when a superconductor is in the Bean critical state, the addition of vortices occurs by increasing the external magnetic field. This procedure is analogous to the introduction of new grains to a sand-pile and is expected to produce an avalanche of grains of sand (or, equivalently, vortices) of all sizes to maintain a constant gradient in the grain (or, magnetic flux) density. We show in this work strong evidences pointing out that, for some specific conditions, magnetic field penetrates 2D-JJA in flux avalanches.
\end{abstract}

\section{Introduction}

Granular superconductors can be considered as a collection of superconducting grains embedded in a weakly superconducting - or even normal - matrix. For this reason, granularity is a term specially related to hightemperature superconductors (HTS), where magnetic and transport properties of these materials are usually manifested by a two-component response. The first one represents the intragranular contribution, associated to the grains exhibiting ordinary superconducting properties. The other component originates from intergranular material, and is associated to the weak-link structure, thus, to the Josephson junctions network. From this picture, intragranular properties would be intrin$s i c$, while intergranular, on the contrary, would be $e x$ - trinsic, generating processing dependent effects. For granular samples in the macroscopic scale, the fraction $f_{g}$, is a measure of the normalized superconducting volume. For single-crystals and other nearly-perfect structures, granularity is a feature that can be considered as the result of a symmetry break. Thus, one might have granularity in the nanometer scale, generated by localized defects like impurities, oxygen deficiency, vacancies, atomic substitutions and the genuinely intrinsic granularity associated with the layered structure of perovskites. On the micrometer scale, granularity results from the existence of extended defects, as grain and twin boundaries. From this picture, granularity could have many contributions, each one with a different volume fraction. In transport and magnetic experiments, this multigranular feature, is recognized through the 
presence of different plateaus ${ }^{[1-4]}$.

The small coherence length of HTS implies that any imperfection may contribute to both the weak-link properties and the flux pinning. This leads to many interesting peculiarities and anomalies, many of which have been tentatively explained over the years in terms of the granular character of HTS materials.

Artificial Josephson junction arrays consist of su- perconducting islands arranged on a symmetrical lattice (Fig. 1), coupled by Josephson junctions, where is possible to introduce a controlled degree of disorder. In this case, a JJA with disorder can be considered as the limiting case of an extreme inhomogeneous type-II superconductor, allowing its study in samples where the disorder is nearly exactly known.
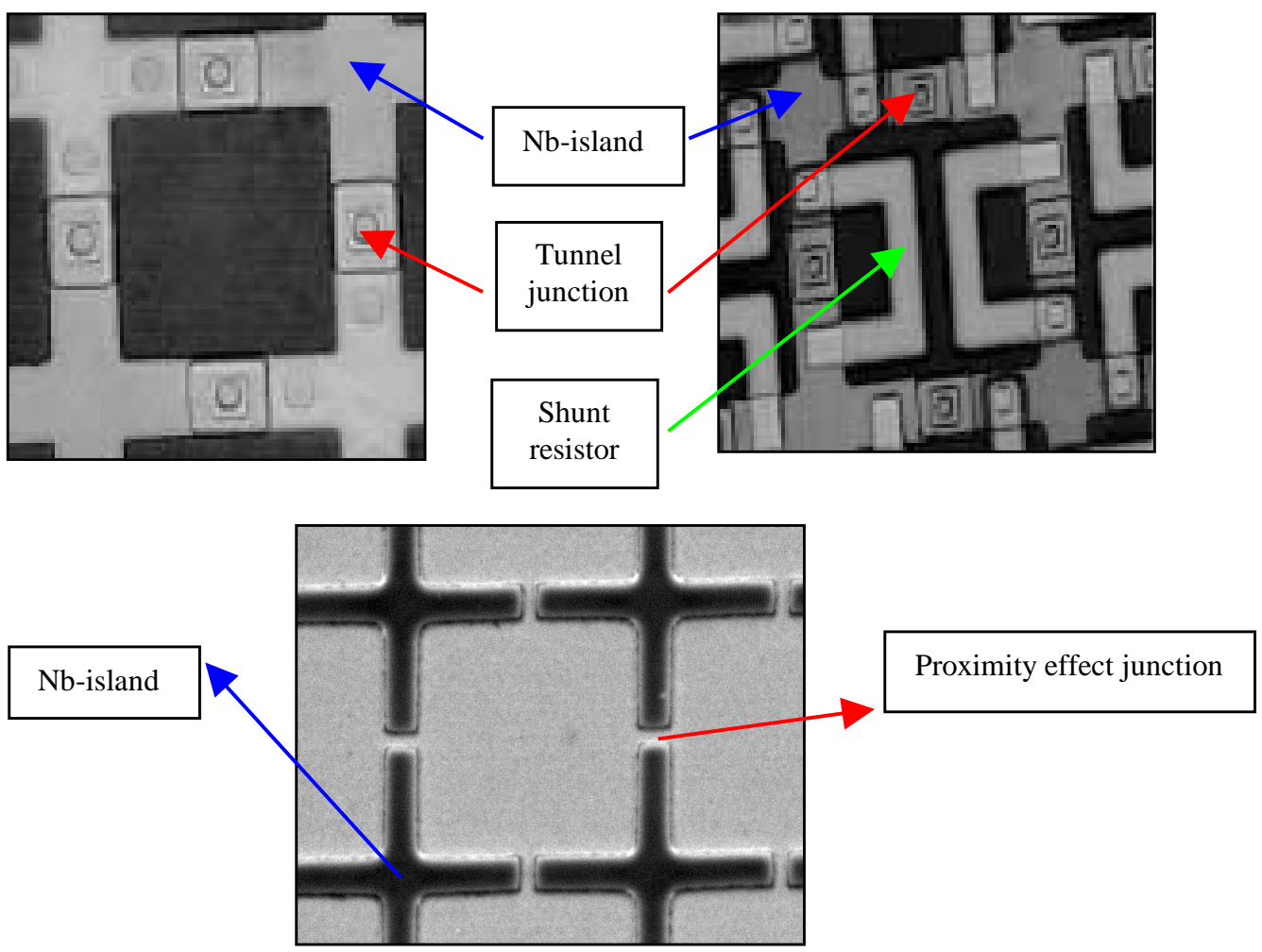

Figure 1. Photographs of Josephson junction arrays of different types: (I) SIS/unshunted, (II) SIS/shunted, and (III) SNS.

Since JJA are artificial structures, they can be very well characterized. Their discrete nature, together with the very well-know physics of the Josephson junction, allows the numerical simulation of their behavior. The details of the physical properties of two dimensional Josephson junction arrays have been recently extensively discussed by Newrock et al.$^{[5]}$, and Martinoli et $a l .{ }^{[6]}$.

In this paper we present our results on the magnetic properties of shunted and unshunted SIS arrays of Josephson junctions (JJA), and their relation to some interesting and intriguing features of granular superconducting systems like the so-called Paramagnetic Meissner Effect (PME) and the influence of the weak link network on its appearance. We also show our recent re- sults related to the occurrence of a critical state (CSM) and the vortex avalanche phenomena (VA), all them observed in granular superconducting systems.

\section{Experimental procedures}

We have performed experiments of $\chi_{A C}(T)$, for different fixed values of $h_{A C}, \chi_{A C}\left(h_{A C}\right)$ for different temperatures (for both experiments $H_{D C}=0$ ) and experiments of scanning SQUID microscopy.

Our samples consisted of $100 \times 150$ shunted and unshunted tunnel junctions, similar to those shown in Fig. 1. The unit cell had square geometry with lattice spacing $a=$ and a junction area of $5 \times 5 \mu \mathrm{m}^{2}$ (Fig. 2). From these dimensions, we estimated that the inductance of 
each loop was about $64 \mathrm{pH}$. The critical current density for the junctions forming the arrays was about 600 $\mathrm{A} / \mathrm{cm}^{2}$ at $4.2 \mathrm{~K}$, giving $I_{C}=150 \mu \mathrm{A}$ for each junction.

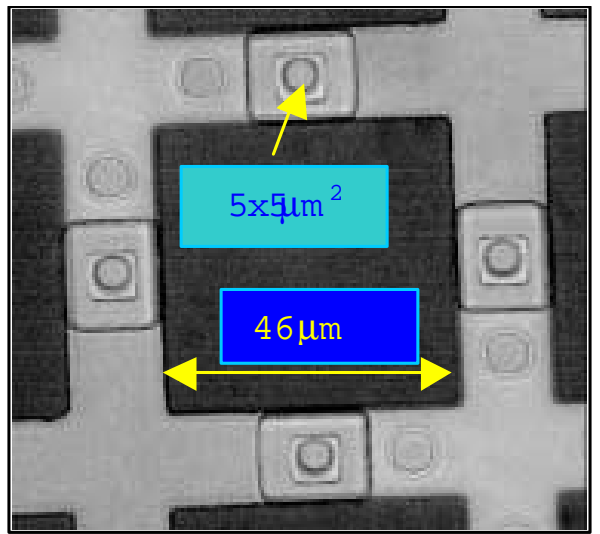

Figure 2. The unit cell of one of our unshunted JJA with square geometry with lattice spacing $a=46 \mu \mathrm{m}$ and a junction area of $5 \times 5 \mu \mathrm{m}^{2}$

\section{(a) AC susceptibility measurements}

Complex AC magnetic susceptibility is a powerful low-field technique to determine the magnetic response of many systems ${ }^{[7]}$, like granular superconductors and Josephson junction arrays. It has been successfully used to measure several parameters such as critical temperature, critical current density and penetration depth in superconductors. To measure samples in the shape of thin films, the so-called screening method has been developed. In the reflection configuration ${ }^{[8]}$, both set of coils (primary and secondaries) are at the same side, and the sample is positioned as close as possible to the set of coils, to maximize the induced signal on the pick up coils (Fig. 3). An alternate current sufficient to create a magnetic field of amplitude $\mathrm{h}_{A C}$ and frequency $f$ is applied to the primary coil. The output voltage of the secondary coils, $V$, is a function of the complex susceptibility, $\chi_{A C}=\chi^{\prime}+\mathrm{i} \chi^{\prime \prime}$, and is measured through the usual lock-in technique. If we take the current on the primary as a reference, $V$ can be expressed by two orthogonal components. The first one is the inductive component, $\mathrm{V}_{L}$ (in phase with the timederivative of the reference current) and the second one the quadrature resistive component, $\mathrm{V}_{R}$ (in phase with the reference current). This means that $\mathrm{V}_{L}$ and $\mathrm{V}_{R}$ are correlated with the average magnetic moment and the energy losses of the sample, respectively. The susceptometer was positioned inside a double wall $\mu$-metal shield, screening the sample region from Earth's magnetic field.

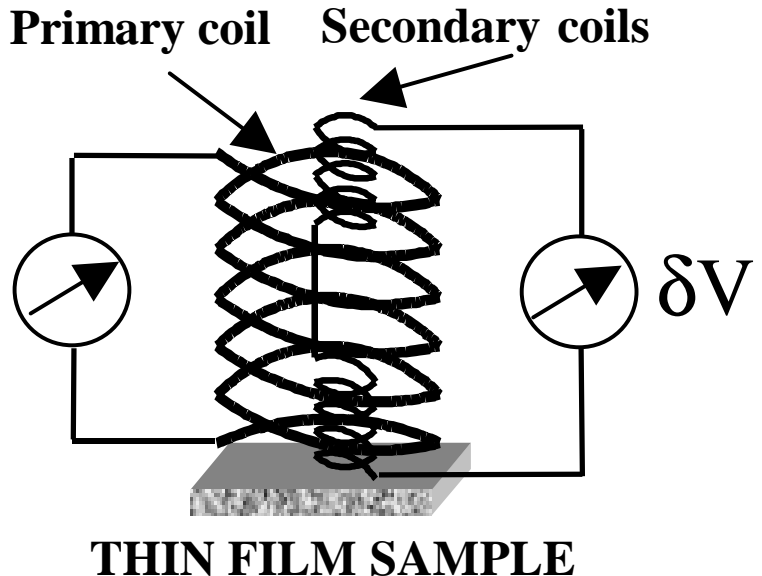

Figure 3. Screening method in the reflection technique, where an excitation coil (primary) coaxially surrounds a pair of counter-wound pick up coils (secondaries).

\section{(b) SSM - Scanning SQUID microscopy}

To study the spatial distribution of magnetic flux in JJA, we have used a scanning superconducting quantum interference device (SQUID) microscope (SSM) using a technique similar to that developed by Kirtley and collaborators ${ }^{[9]}$. The SSM provides spatially resolved magnetization images of the sample. The sample is uniformly attached to the thermal bath, eliminating thermal gradients across the sample during cooling.

The array is placed in a SSM sample stage ${ }^{[10]}$ sitting at the end of a sapphire rod of $10 \mathrm{~mm}$ diameter, around which a solenoid has been. To avoid interaction with sample, and before collecting data, we removed possible magnetic flux trapped within the pick up loop of the SQUID ${ }^{[11]}$. Also, the magnetic field background has been measured and corrected.

The basic experimental procedure consists in cooling down the sample to $4.2 \mathrm{~K}$ following two routes: (a) with no applied magnetic field (ZFC experiments), and (b) in a fixed external magnetic field (FC experiments). After the sample reached the final temperature, we use the SSM to measure the magnetic flux threading the SQUID from the sample, $\Phi_{T O T}$, as a function of the position. The spatial resolution of the SSM is limited by the separation distance SQUID-sample, which has been estimated to be in the interval 40-60 $\mu \mathrm{m}$. The external magnetic flux, $\Phi_{E X T}$, is directly determined from an SSM image of the sample at a temperature above $\mathrm{T}_{C}$. 


\section{Numerical simulations}

We have found that all the experimental results obtained from the magnetic properties of JJA can be qualitatively explained by analyzing the dynamics of a single unit cell in the array ${ }^{[12,13]}$.

In our experiments, the unit cell is a loop containing four junctions and the measurements correspond to ZFC AC magnetic susceptibility. We model a single unit cell as having four identical junctions, each with capacitance $C_{J}$, quasi-particle resistance $R_{J}$ and critical current $I_{C}$. We apply an external field of the form:

$$
H_{e x t}=h_{A C} \cos (\omega t)
$$

In the case of an oscillatory external magnetic field of the form of Eq. [1], the magnetization is given by:

$$
M=\frac{L I}{\mu_{0} a^{2}}
$$

Here, $\mu_{0}$ is the vacuum permeability, $I$ is the circulating current in the loop, $L$ is the inductance of the loop and a is the lattice spacing. M may be expanded as a Fourier series in the form:

$$
M(t)=h_{A C} \sum_{n=0}^{\infty}\left[\chi_{n}^{\prime} \cos (n \omega t)+\chi_{n}^{\prime \prime} \sin (n \omega t)\right]
$$

We calculated $\chi^{\prime}$ and $\chi^{\prime \prime}$ through this equation. Both Euler and fourth-order Runge-Kutta integration methods provided the same numerical results. In our model we do not include other effects (such as thermal activation) beyond the above equations. In this case, the temperature-dependent parameter is the critical current of the junctions, given to good approximation by ${ }^{[14]}$ :

$$
I_{C}(T)=I_{C}(0) \sqrt{1-\frac{T}{T_{C}}} \tanh \left[1.54 \frac{T_{C}}{T} \sqrt{1-\frac{T}{T_{C}}}\right]
$$

We calculated $\chi_{1}$ as a function of $T . \chi_{1}$ depends on the parameter $\beta_{L}$, which is proportional to the number of flux quanta that can be screened by the maximum critical current in the junctions, and the parameter $\beta_{C}$, which is proportional to the capacitance of the junction:

$$
\begin{aligned}
& \beta_{L}(T)=\frac{2 \pi L I_{C}(T)}{\Phi_{0}} \\
& \beta_{C}(T)=\frac{2 \pi I_{C} C_{J} R_{J}^{2}}{\Phi_{0}}
\end{aligned}
$$

By using the equations above, we can simulate the magnetic behavior of a particular JJA with specific parameters $\beta_{C}, \beta_{L}$, and $\mathrm{I}_{C}(\mathrm{~T})$. This last parameter gives the temperature dependency of the simulated properties.

\section{Results and discussion}

\section{(a) The Paramagnetic Meissner Effect}

The paramagnetic Meissner effect (PME) measured in high $\mathrm{T}_{C}$ granular superconductors ${ }^{[15]}$ has been attributed to the presence of $\pi$-junctions between the grains ${ }^{[16]}$. Here we present measurements of complex AC magnetic susceptibility from two-dimensional arrays of conventional (non $\pi$ ) $\mathrm{Nb}-\mathrm{AlO}_{x}-\mathrm{Nb}$ Josephson junctions. We measured the AC susceptibility as a function of the temperature $T$, the $\mathrm{AC}$ amplitude of the excitation field, $h_{A C}$, and the external magnetic field, $H_{D C}$. The experiments show a strong paramagnetic contribution from any multi-junctions loop, which manifests itself as a reentrant screening at low temperature, for values of $h_{A C}$ higher than 50 mOe. The highly simplified model described before, based on a single loop containing four junctions, accounts for this paramagnetic contribution and the range of parameters in which it appears. This model offers an alternative explanation of PME which does not involve $\pi$-junctions. This paramagnetic response was in striking contrast to the usual diamagnetic Meissner effect, where the magnetic field is excluded from superconductors. The PME appeared systematically under specific experimental conditions and depended on sample preparation and morphology. Braunisch et al. ${ }^{[15]}$ found that, after cooling the same samples following a zero external field procedure (ZFC), the measured susceptibility was diamagnetic. The authors attributed PME to the occurrence of spontaneous currents, flowing in direction opposite to ordinary Meissner screening currents. They proposed that anomalous Josephson junctions between the grains may be responsible for the existence of such currents. In these junctions ( $\pi$-junctions) the Cooper pairs acquire a phase shift equal to $\pi$ in the tunneling process and the Josephson current has direction opposite to conventional junctions. $\pi$-junctions may be the consequence of magnetic impurities in the junction, or non-s wave pairing symmetry ${ }^{[16]}$.

Fig. 4 shows the results for $\chi_{A C}(T)$, obtained from ZFC experiments, for different amplitudes of the AC magnetic field. For $h_{A C}$ smaller than about $50 \mathrm{mOe}$, the behavior of both components of $\chi(T)$ is quite similar to typical superconducting samples. The real component, $\chi^{\prime}(T)$, which is a measure of the screening current, becomes more negative at lower temperatures, indicating stronger superconductivity through the Meissner effect. On the other side, the imaginary compo- 
nent $\chi "(T)$ peaks, indicating a maximum in the losses, around the critical temperature, $\mathrm{T}_{C}$. Notice that $\chi^{\prime} \approx-$ 0.7 (SI) for $h_{A C}=10 \mathrm{mOe}$, at low temperature. The sample can only partially screen the external magnetic field. Outside the Meissner-like regime, for values of $h_{A C}>50 \mathrm{mOe}, \chi^{\prime}(T)$ is reentrant. It first increases in modulus as the temperature is lowered from the critical temperature $\mathrm{T}_{C}$, then decreases at a lower temperature. The minimum in $\chi^{\prime}(T)$ appears at $\mathrm{T} \approx 7.0 \mathrm{~K}$. For all the temperatures, at a fixed value of $\mathrm{T}$, the modulus of $\chi^{\prime}(T)$ decreases by increasing $h_{A C}$. The out-of-phase component, $\chi$ " $(T)$, is correlated with the reentrance observed in $\chi^{\prime}(T)$, showing increasing losses as the screening decreases, indicating an apparent weakening of the order parameter at low temperatures.
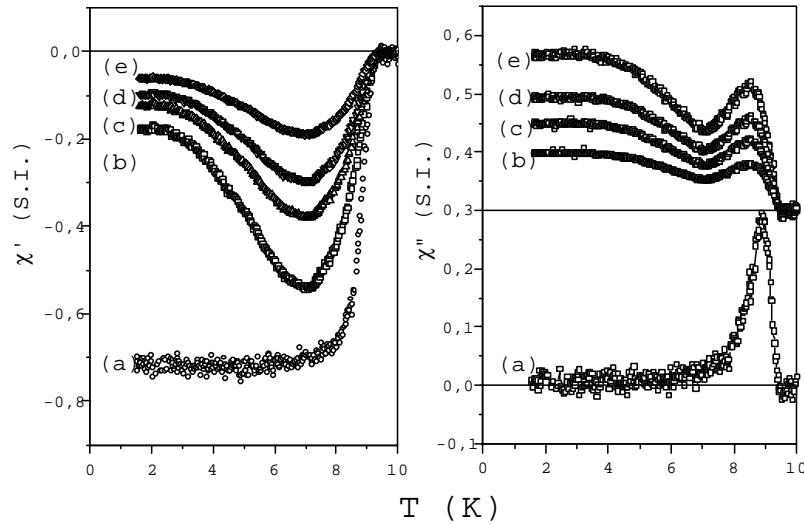

Figure 4. Curves of $\chi_{A C}(T)$ for (a) $h_{A C}=10$, (b) $h_{A C}=80$, (c) $h_{A C}=96$, (d) $h_{A C}=144$ and (f) $h_{A C}=320$ mOe with no applied external magnetic field.

We have simulated $\chi_{1}^{\prime}(T)$ and $\chi_{1} "(T)$ for different values of $h_{A C}$ by following the equations developed before. The obtained results are shown in Fig. 5. For values of $h_{A C}$ smaller than $47 \mathrm{mOe}$ (corresponding to $\left.\mu_{0} a^{2} h_{A C} \approx 5 \Phi_{0}\right), \chi_{1}^{\prime}(T)$ decreases with decreasing temperature, and $\chi_{1} "(T)$ is close to zero. By increasing $h_{A C}$ above $47 \mathrm{mOe}$, reentrance at low temperature clearly appears and the screening becomes weaker in all the temperature range. This is consistent with the experiment, where the magnitude of $\chi^{\prime}(T)$ decreases with increasing $h_{A C}$. Note that at these high values of $h_{A C}$ the simulated $\chi_{1}$ " $(T)$ increases significantly, i.e. the simulation reproduces the dramatic increase of losses at low temperature that is found in the experiment.

Thus, phenomena causing the reentrance we observed in Josephson junction arrays should also exist in granular superconductors. This has been recently experimentally reported by Passos et al. ${ }^{[17]}$ where HTS samples with controlled granularity, have shown reentrance. The reentrance behavior, as expected from our work, appears either as PME, in the case of DC susceptibility measurements, or as an anomalous increase of dissipation at low temperature, in the case of AC susceptibility measurements. Numerical simulations of two-dimensional Josephson junction networks with a distribution of characteristic parameters $\beta_{L}$ and $\beta_{C}$ would be very useful for further theoretical investigations of these phenomena, as we explore in the next Section.


Figure 5. Curves for the simulated $\chi_{1}^{\prime}(T)$ and $\chi_{1} "(T)$ for (a) $h_{A C}=5 \mathrm{mOe}$, (b) $29 \mathrm{mOe}$, (c) $69 \mathrm{mOe}$, (d) $118 \mathrm{mOe}$. In all cases $\beta_{L}(T=4.2 K)=30$ and $\beta_{C}(T=4.2 K)=60$. Curves for $\chi_{1}$ " $(T)$ have been vertically shifted by 0.4 (SI) for clarity.

\section{(b) The Stewart-McCumber parameter and the occurrence of PME}

In the preceding section we show that the Paramagnetic Meissner Effect is directly associated to pinning, and not necessarily related to the presence of $\pi$-junctions. However, it is still unclear which are the parameters controlling the occurrence of PME in granular systems. Besides those works from Braunisch and co-workers mentioned before, PME has also been observed in certain samples of $\mathrm{YBa}_{2} \mathrm{Cu}_{3} \mathrm{O}_{7-d}^{[18]}$, and $\mathrm{Nd}_{2-x} \mathrm{Ce}_{x} \mathrm{CuO}_{y}^{[19]}$. The parameters to control the appearance of the PME in granular systems are still to be determined. One of the main problems in controlling $\mathrm{PME}$ is the difficulty to reproduce identical parameters in different samples, even following the same preparation procedure. Among samples processed in identical way, just a few of them show PME. This is consistent with experimental evidences indicating that this effect is directly related to the weak link network present in all granular systems ${ }^{[15]}$. Many authors have verified that the surface of the sample plays an important roll in the appearance of $\mathrm{PME}^{[20]}$. Both, the weak link network and the sample surface are, up to now, hardly reproducible from one sample to another in a sufficient way to guarantee the control of all the properties, including the appearance of the PME. Other authors have proposed alternative models to explain it, based on flux trapping effects. PME can exist as a consequence of pinning, and that $\pi$-junctions - resulting from the presence of 
magnetic impurities in the junction or non-s-wave symmetry are not necessarily related to the occurrence of this effect. We have shown that PME manifests itself as a reentrance of the $\mathrm{AC}$ magnetic susceptibility as a function of temperature. One unsolved question still remains within the scenario we have previously proposed: why the dynamics reentrance in $\chi_{A C}(T)$ has never been measured in standard-prepared high temperature superconductors? We propose an answer to this question, showing that, among the systems exhibiting PME, only those with sufficiently low dissipation and high capacitance will show dynamics reentrance.

As we have shown PME manifests itself in twodimensional unshunted Josephson junction arrays as reentrance in the temperature dependent $\mathrm{AC}$ magnetic susceptibility. However, reentrance is not observed in shunted arrays, where the value of the StewartMcCumber parameter, $\beta_{C}$, is very low compared to unshunted arrays. We have observed this absence of the reentrant behavior in three sets of shunted samples with different parameters and geometries.

The reentrance in the $\mathrm{AC}$ magnetic susceptibility is extremely unusual in granular systems whose weak links have a broad distribution of their characteristic parameters. The dependence of the reentrance on the value of $\beta_{C}$ is consistent with the systematic of PME in granular superconductors. This result explains why reentrance - therefore PME - is hardly observed in granular superconductors besides those with controlled granularity. These experimental findings are confirmed by numerical simulations of the same simplified model based on a loop containing four junctions.

To verify the influence of the parameter $\beta_{C}$ on the reentrance, we have performed numerical simulations of the same four junctions model previously described. Again, as in the PME situation, this model gives an excellent semi-quantitative description to all our experimental findings.

We show the results of the simulated $\chi_{1}(T)$, corresponding to $\beta_{C}(T=4.2 K)=30$ (unshunted arrays) and $\beta_{C}(T=4.2 K)=1$ (shunted arrays). In all numerical simulations we have used $\beta_{L}(T=4.2 K)=30$, in agreement with the real values of these parameters for both types of samples. From the definition of these parameters, we can see that even for the case of high values of the critical current $I_{C}$, very low values of the resistance and the capacitance of the junction $\left(\mathrm{R}_{J}\right.$ and $\mathrm{C}_{J}$, respectively), can determine low values of $\beta_{C}$. Typical behavior of this parameter in two extreme conditions are $\beta_{C}=0$ and $\beta_{C} \rightarrow \infty$, corresponding to negligible and dominant capacitance, respectively. The parameter $\beta_{C}$ can be associated to the quality of the weak link, i.e. the Josephson junction. Therefore, a Josephson junction with a low $\beta_{C}$ value, is associated to a lowquality weak link, and can be modeled by a Josephson junction in parallel with a resistor $\mathrm{R}$. In spite of $\beta_{C}$ and $\beta_{L}$ have never been determined for granular supercon- ductors, their $\mathrm{I} \times \mathrm{V}$ characteristics show that they can be considered as low- $\beta_{C}$ systems ${ }^{[21-23]}$.

To further understand the influence of the different values of $\beta_{C}$ in the reentrance, it is essential to consider the curve $\Phi_{T O T}$ vs. $\Phi_{E X T}$ for a shunted sample (Fig. 6). Similarly to unshunted samples ${ }^{[12,13]}$, for a fixed temperature, this curve is very hysteretic, showing multiples branches intersecting the line $\Phi_{T O T}$ $=0$ which corresponds to diamagnetic states. For all the other branches, their intersection with the line $\Phi_{T O T}=\Phi_{E X T}$ corresponds to the boundary between diamagnetic states (negative values of $\chi^{\prime}(T)$ ) and paramagnetic states (positive values of $\chi^{\prime}(T)$ ), as explained in Section V. For unshunted arrays, at the temperatures of $\mathrm{T}=5 \mathrm{~K}$ and $\mathrm{T}=7.6 \mathrm{~K}$, the appearance of the second branch and the third branches respectively add a paramagnetic contribution to the average value of $\chi^{\prime}(T)$. The analysis of these figures, shows why there is no reentrance in low temperatures when $\beta_{C}$ is small. In this case of low $\beta_{C}$, the second branch appears to be stable, giving an extra diamagnetic contribution overwhelming the paramagnetic contribution from subsequent branches. When $\beta_{C}$ is large (unshunted arrays case), the second branch is unstable, and the average response of the sample in low temperatures is paramagnetic.

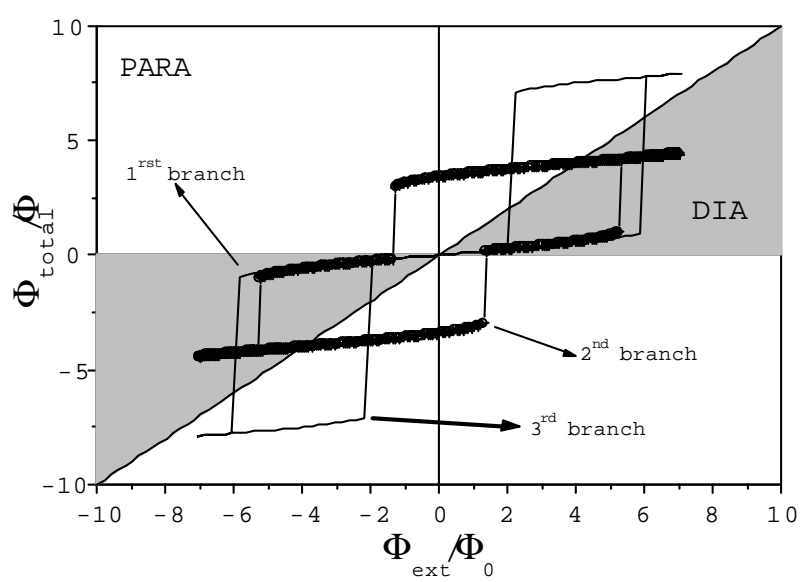

Figure 6. Numerical results for the simulation of $\Phi_{T O T}$ vs. $\Phi_{E X T}$ for a fixed temperature.

For unshunted arrays, PME occurs when some weak links have a sufficiently large critical current. Therefore, the effect occurs above a corresponding minimum value of $\beta_{L}$. However, experiments show and simulations confirm that, even for large values of $\beta_{L}$, reentrance does not occur when $\beta_{C}$ is of the order of 2 or smaller. In this case, the curve $\Phi_{T O T}$ vs. $\Phi_{E X T}$ is very hysteretic, appearing extra branches giving an average diamagnetic behavior. For low $\beta_{C}$, when the samples are ZFC and then measured at small values of the magnetic field, most of the loops will be in states corresponding to the diamagnetic states, and no paramagnetic response can be measured. Even when the 
samples are FC in small magnetic fields, flux quanta can not get trapped into the loops. In this situation, the sample remains diamagnetic even at higher values of the excitation magnetic field. When PME does not appear as a consequence of low values of $\beta_{C}$, there is no crossover from paramagnetic to diamagnetic response by increasing the applied magnetic field, as we measured for the unshunted arrays. Thus, the grains and the junctions will have the same diamagnetic response and the measured value of the magnetic susceptibility will be negative, i.e., diamagnetic. (a)

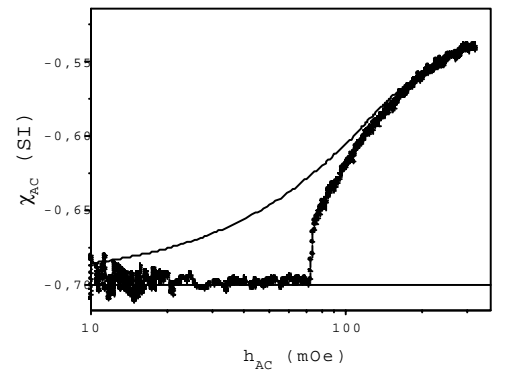

(b)

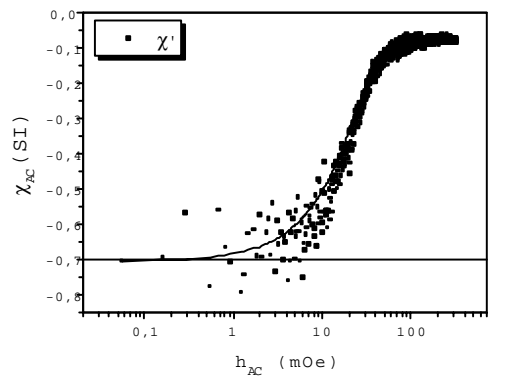

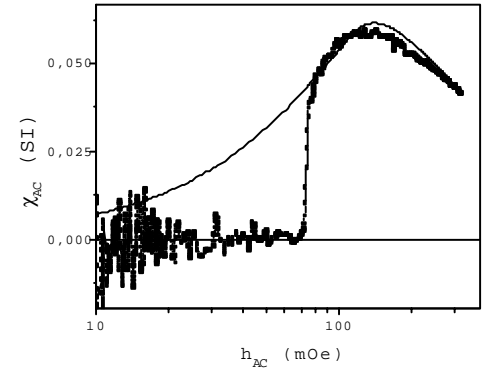

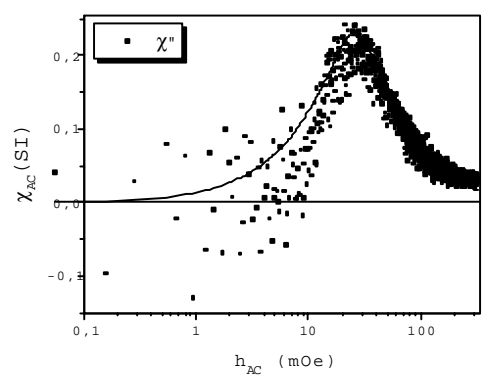

Figure 7. Curves for $\chi\left(h_{A C}\right)$, for both components of the AC magnetic susceptibility; the continuous line is the fitting to the exponential critical state model (a) $\mathrm{T}=4.2 \mathrm{~K}$, (b) $\mathrm{T}=8.0$.

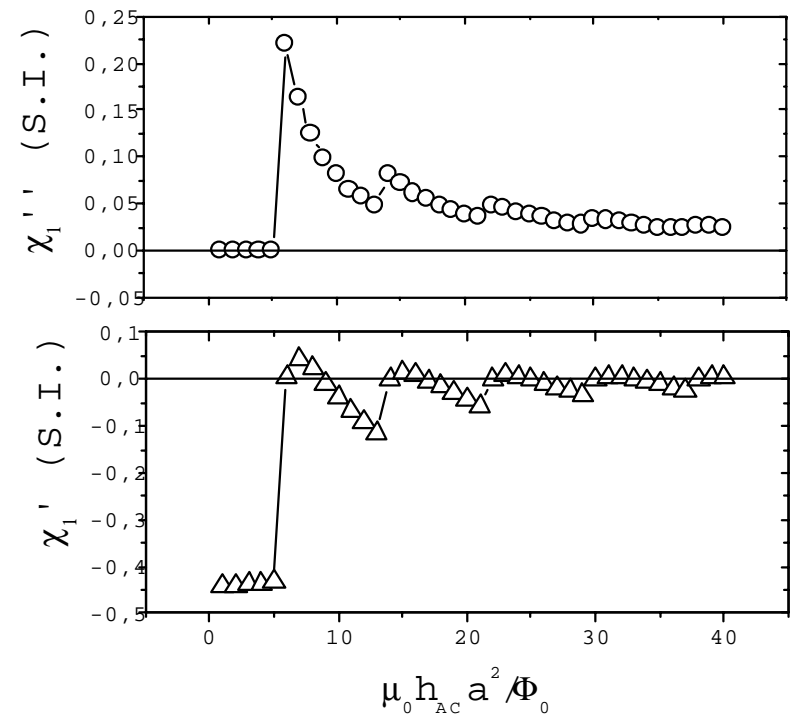

Figure 8. Numerical result for $\chi\left(h_{A C}\right)$ for $\mathrm{T}=4.2 \mathrm{~K}$, using the four-junctions model. As can be observed, there is a sharp increase in both $\chi^{\prime}$ and $\chi^{\prime \prime}$, around $h_{A C}=50 \mathrm{mOe}$
Thus, we have shown that only high values of the critical current of the weak links are not enough to cause reentrance (i.e., PME) in agreement with the experimental results proposed by other groups. Our results also indicate that the dissipation and the capacitance of the weak links, and not only the inductance, play the more important role in the appearance of the reentrance. We have also verified that, even for large values of $\beta_{L}$, reentrance does not occur if $\beta_{C}$ is very small. This explains why reentrance is uncommon and hardly reproducible in granular high temperature superconductors, since these materials are associated to low values of the parameter $\beta_{C}$, when prepared by following standard procedures. All our experimental findings are in perfect agreement with the results obtained numerically from the simple model based on a loop containing four junctions. It is straightforward to conclude that, controlling the weak link coupling in granular superconducting samples, would be possible to control the appearance of the reentrance, and, in consequence, the appearance of PME. 


\section{(c) Critical state model in 2D-JJA}

The concept of a critical-state and its use in the interpretation of $\mathrm{AC}$ magnetization data in terms of a critical current density were introduce by both Bean $[24,25]$ and London ${ }^{[26]}$ in a successful effort to derive the magnetic properties of hard type-II superconductors. However, the relation between local and global quantities is more complex in HTS than in LTS. Bean's original model is inadequate for polycrystalline HTS superconductors, manly because of their strong magnetic field dependence of the critical current which leads to current limitation in a macroscopic wire caused by the magnetic self-field ${ }^{[27]}$. The critical-state model proposed by Bean provides a mechanism for harmonic generation in the components of the magnetization of typeII superconductors, when immersed in an AC magnetic field.

Different critical state models ${ }^{[27-29]}$ assuming that supercurrents flow inside the sample with a critical density $J_{C}\left(H_{i}\right)$ (where $H_{i}$ is the internal magnetic field), have been used to study the magnetic response of typeII superconductors to an external field [30-35]. In particular, Chen et al..$^{[31]}$ have shown that the exponential law, first introduced by Fietz et al. ${ }^{[29]}$, is very useful to interpret AC susceptibility data of HTS.

In spite of all the numerical evidence bringing out the equivalence between critical-states in JJA and HTS, to our knowledge there are no direct experimental proofs supporting such relation. In this Section, we present our numerical and experimental results studying the penetration of the magnetic field and its relation with a critical-state in unshunted two-dimensional Josephson junction arrays. We have numerically simulated the dependence of $\chi^{\prime}$ and $\chi$ " on $h_{A C}$. We have obtained experimental results by using two techniques, mutual-inductance measurements, and scanning SQUID microscopy.

From the mutual-inductance experiments, we have obtained the AC magnetic susceptibility as a function of the $\mathrm{AC}$ magnetic field, $\chi\left(h_{A C}\right)$, for different temperatures. In these experiments, we keep constant the temperature and the external DC magnetic field, and sweep the AC magnetic field, $h_{A C}$. Fig. (7a-b) shows the measured $\chi_{A C}(T)$ vs. $h_{A C}$ in our arrays, at $\mathrm{T}=$ $4.2 \mathrm{~K}$ and $\mathrm{T}=8.0 \mathrm{~K}$. We observe that there is a sharp increase in both $\chi^{\prime}$ and $\chi^{\prime \prime}$, around $h_{A C}=50 \mathrm{mOe}$. The amplitude of this jump decreases as we increase the temperature, until $\mathrm{T} \approx 5.0 \mathrm{~K}$. For $T>5 \mathrm{~K}$ there is no longer a discontinuity. This discontinuity in the curve is a signature of the transition from the Meissner-like regime to the reentrant regime.

We have also numerically determined the dependence of $\chi^{\prime}$ and $\chi$ " on $h_{A C}$, by considering the fourjunctions model described in previous sections. In this case we have used $\beta_{L}=30$ e $\beta_{C}=60$.This results is qualitatively in agreement with that shown in Fig. 8.

To study the distribution of magnetic flux in our unshunted SIS samples, we have performed several experiments by using a scanning SQUID microscope, as described before.

In the first SSM experiment (Fig. 9), we induce flux into the array using the feedback coil patterned on the SQUID chip. The sample was heated above $\mathrm{T}_{C}$, a small current was put through the feedback coil, and the sample was cooled to $4.2 \mathrm{~K}$. Then we heated up the sample and observed how the trapped flux behaved as it warmed. The $y$-axis is about $0.5 \mathrm{~mm}$ long and the $\mathrm{x}$-axis is about $1.5 \mathrm{~mm}$ long. The SQUID output is in volts from the electronics. To get the normalized flux in the array, the conversion is: 1.72 volts $=1$ flux quantum in SQUID and 1 flux quantum in SQUID = $(4.7)^{2}=22.09$ flux quanta in the array. From images shown in Fig. 9, we observed no order in the magnetic flux distribution, as we expect for a critical state as we increase the temperature.

In the second SSM experiment (Fig. 10) we made images of successive scans of a large area of the sample, for $\mathrm{T}=4.2 \mathrm{~K}$, as the external magnetic field is increased. These images show that for large areas, the magnetic field penetrates via dendritic growth, as observed by Durán et al ${ }^{[36]}$.

In the third SSM experiment (Fig. 11), the image was taken from a small section of the sample. It was zero field cooled (ZFC), and external flux is zero. The scale on the image is normalized to $\Phi / \Phi_{0}$ in the array, where the $x$ and $y$ axes are in millimeters. From this image, we observed the typical order in the magnetic flux distribution, as we expect for a critical state.

Thus, we have shown that, in principle, there is some long-range order in the distribution of magnetic flux, as required to establish a critical state. As predicted by Chen et al., first it is established a vortex state which changes to a critical state as temperature (or, alternatively, the magnetic field) is increased. From large images, we have observed that magnetic flux penetrates de samples following dendritic growing, as established before by Durán et al. for Nb thin films, using a magneto-optical technique.

\section{(D) Vortex avalanches in 2D-JJA}

The magnetization properties of type-II superconductors have been studied for many decades. The interest about this problem has been renewed after the discovery of HTS. Magnetic flux penetrates these materials in the form of quantized vortices that move via over-damped dynamics. They are subjected to repulsive interactions from other vortices and to random pinning forces due to the inhomogeneities always present in polycrystalline samples. Vortices can move when an electrical current is applied and their movement creates an electrical resistance that can destroy the superconducting state. In this way, structural defects can pin vortices avoiding the material becoming normal. 
(a)

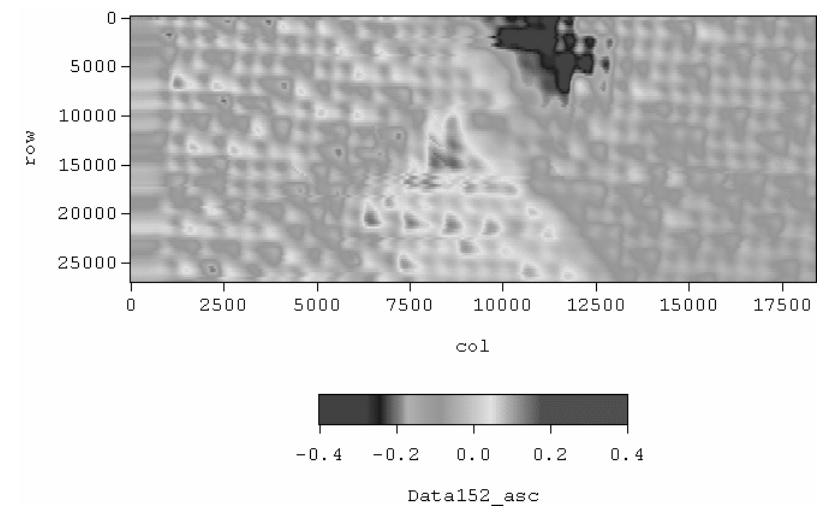

(b)

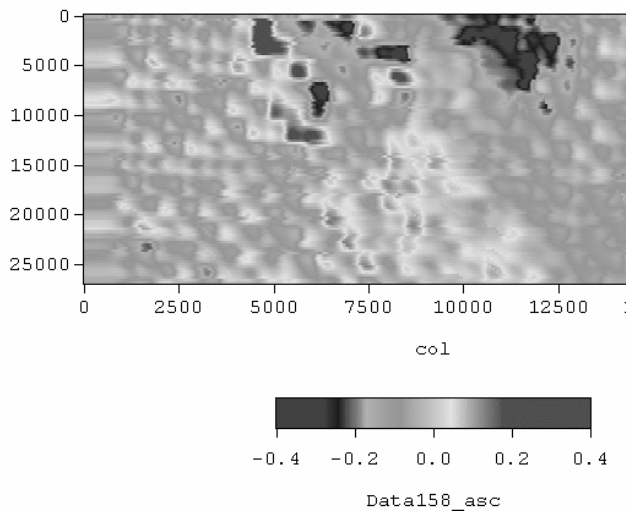

(c)



Figure 9 - SSM images for (a) T=4.2 K, (b) $\mathrm{T}=7.0 \mathrm{~K}$, and (c) $\mathrm{T}=8.0 \mathrm{~K}$

The magnetization process is usually described in terms of a critical-state model providing a consistent picture about the average magnetization properties such as the hysteresis loop and thermal relaxation effects ${ }^{[28]}$. In Bean's model, flux lines penetrate into the sample and, due to the presence of disorder, they give rise to a steady flux gradient. Therefore, the distribution of vortices entering a superconductor is not homogeneous and the finite gradient in the vortices density builds up to create a driving force inward balanced by pinning forces opposing vortices movement. De Gennes ${ }^{[37]}$ originally compared the slope of this pile of vortices to a sand-pile, with the slope being proportional to the local magnitude of the critical current. Dynamic properties of the sand-pile problem have attracted new attention since it consists of a marginally stable system displaying self-organized criticality (SOC) ${ }^{[38]}$. In this case, when a superconductor is in the Bean critical-state, the addition of vortices occurs by increasing the external magnetic field. This procedure is analogous to the introduction of new grains to a sand-pile and is expected to produce an avalanche of grains of sand (or, equivalently, vortices) of all sizes to maintain a constant gradient in the grain (or, magnetic 
(a)



mm

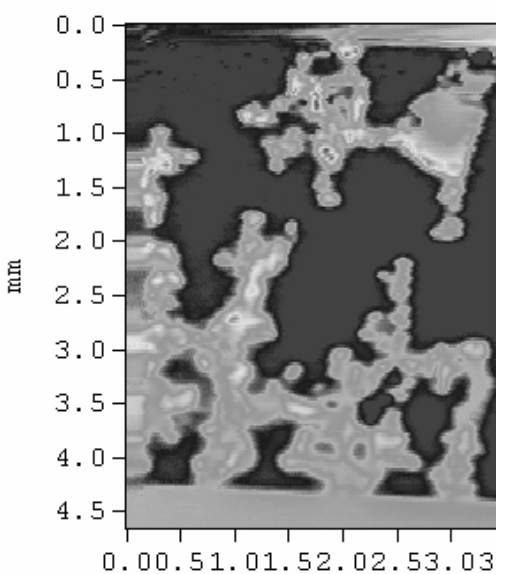

mm

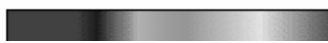

Figure 10. Large sweep SSM images as the external magnetic field is increased for $\mathrm{T}=.2 \mathrm{~K} ; x$ and $y$ axes are in millimeters, for (a) $\mathrm{I}_{\text {coil }}=0.10 \mathrm{~mA}$, and (b) $\mathrm{I}_{\text {coil }}=0.25$ $\mathrm{mA}$.

flux) density. However, Bean's model does not account for those local spatio-temporal fluctuations. Recently, it has been experimental and numerically verified that vortices can move intermittently in time as they are forced in or out of a superconductor ${ }^{[39-41]}$ (as the avalanches mentioned before), and that flux fronts are not smooth ${ }^{[42-44]}$.

The interest in the dynamics of systems that can be slowly driven to the threshold of instability, like the sand-pile, has been increased over the years. This has

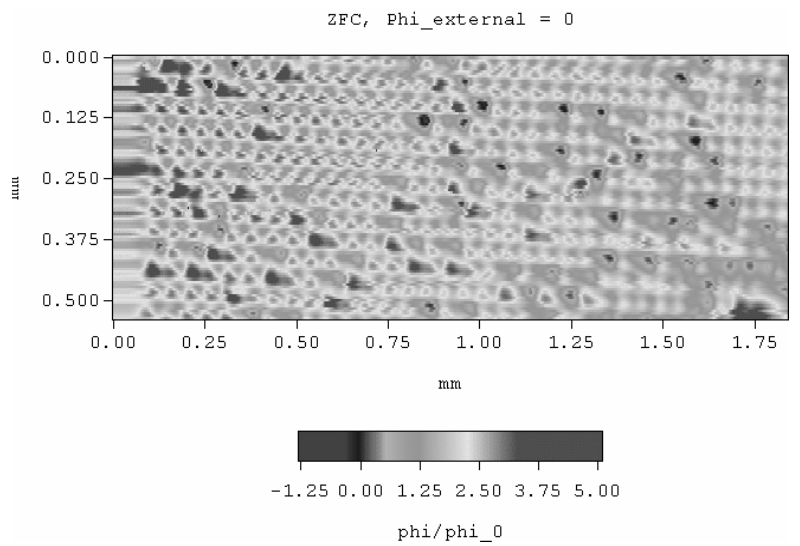

Figure 11. SSM images of a small section of the sample after ZFC and for no external magnetic flux. Dimensions of scanned area are shown in millimeters.

occurred mainly because of the large number and diversity of physical systems characterized by such dynamics, where we can include charge-density waves, pinned Wigner crystals, earthquake faults, granular assemblies, besides those related to superconducting vortices, as shortly described before. However, to experimentally study vortex avalanche phenomena, good quality samples and extremely low temperatures (of the order of $\mathrm{mK})$ are necessary. On the other side, to perform the same study by using numerical procedures, like molecular dynamics or Monte Carlo methods, large machine times are necessary. It would be of great interest if vortex avalanche phenomena could be study in highlycontrolled artificial structures like Josephson junction arrays, whose study is much simpler than those mentioned before.

Experimental data showing that unshunted Josephson junction arrays exhibit vortex avalanche-like phenomena. We show in Fig. 12 AC magnetic susceptibility measurements that, present a succession of steps as temperature is increased. These steps appear before the reentrant behavior well described in preceding sections. We can observe in Fig. 13 that these steps are equally spaced in temperature. As the temperature is raised up, the critical current of the junctions changes accordingly to eq. [4]. Therefore, the mechanism associated to the appearance of the steps seems to be related to a matching between the present value of the parameter $\beta_{L}$ (which also depends on the critical current, so the temperature, as stated by eq. 5) and the available external magnetic field. The $\beta_{L}$ parameter can be described as the capacity of the system (or, the critical current of the array) to screen the external magnetic field. Thus, when this matching happens, the sample lets magnetic flux to enter the sample, following a catastrophic way. 


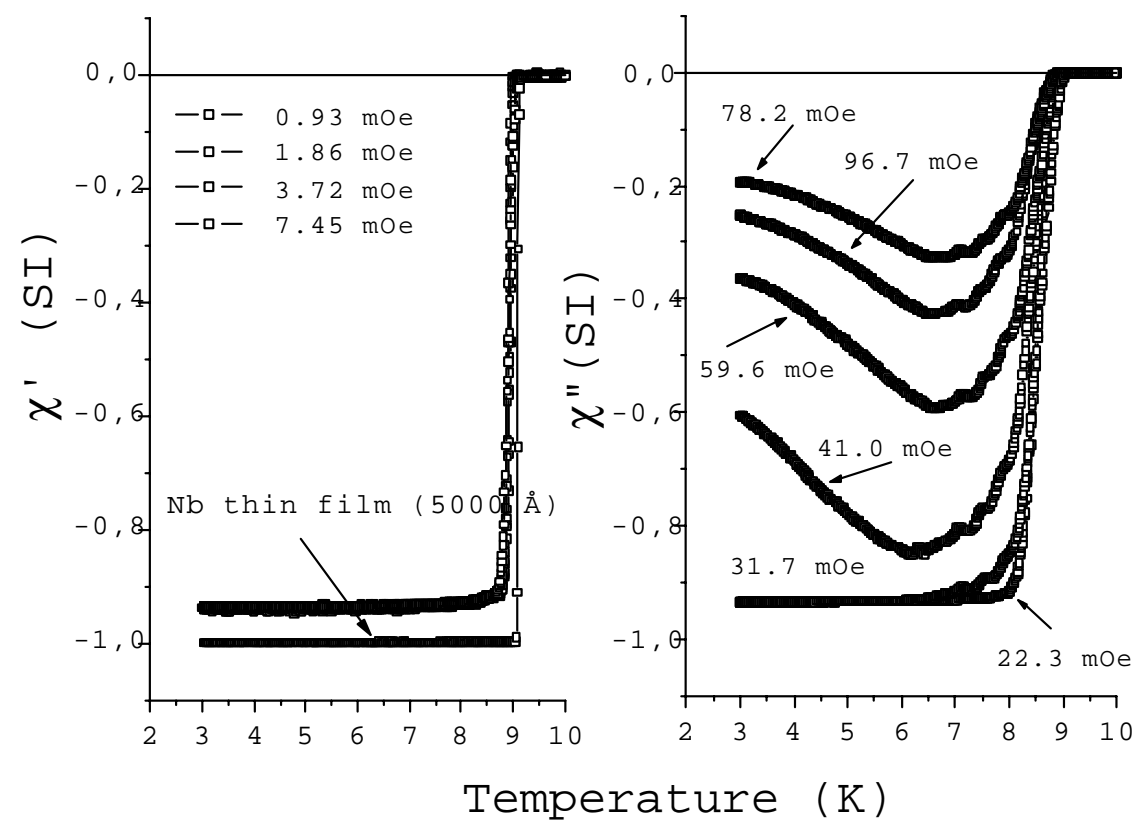

Figure 12. AC magnetic susceptibility measurements for different excitation fields, obtained from an unshunted JJA, showing a succession of steps as the temperature is increased.

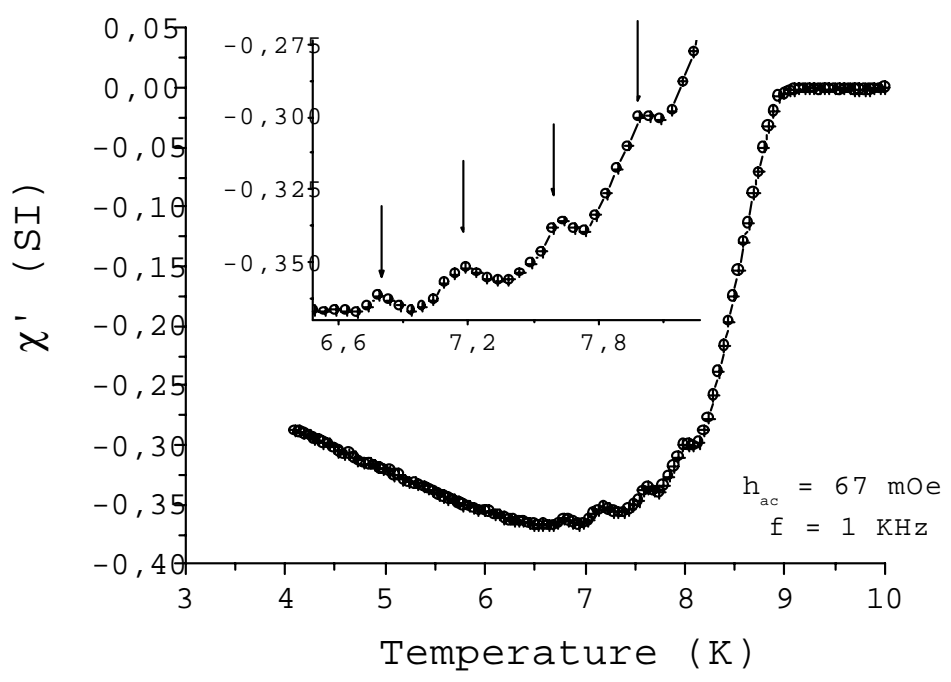

Figure 13. Real component of the AC magnetic susceptibility as a function of temperature, showing the equally-spaced steps (see inset). 
(a)
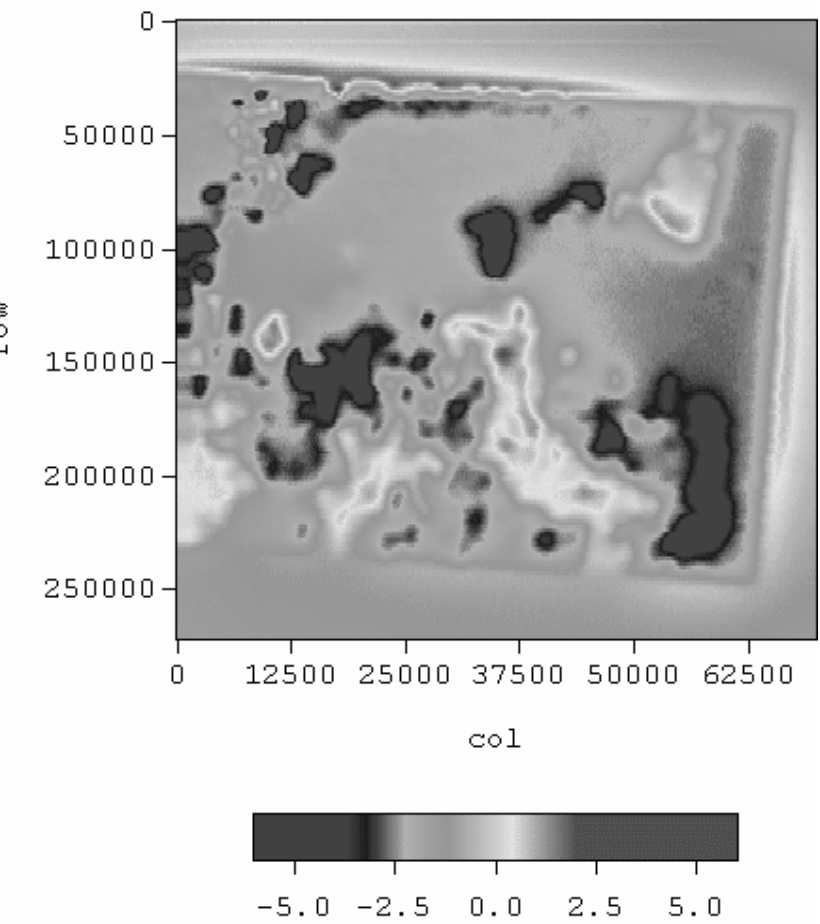

Sweep0 asc

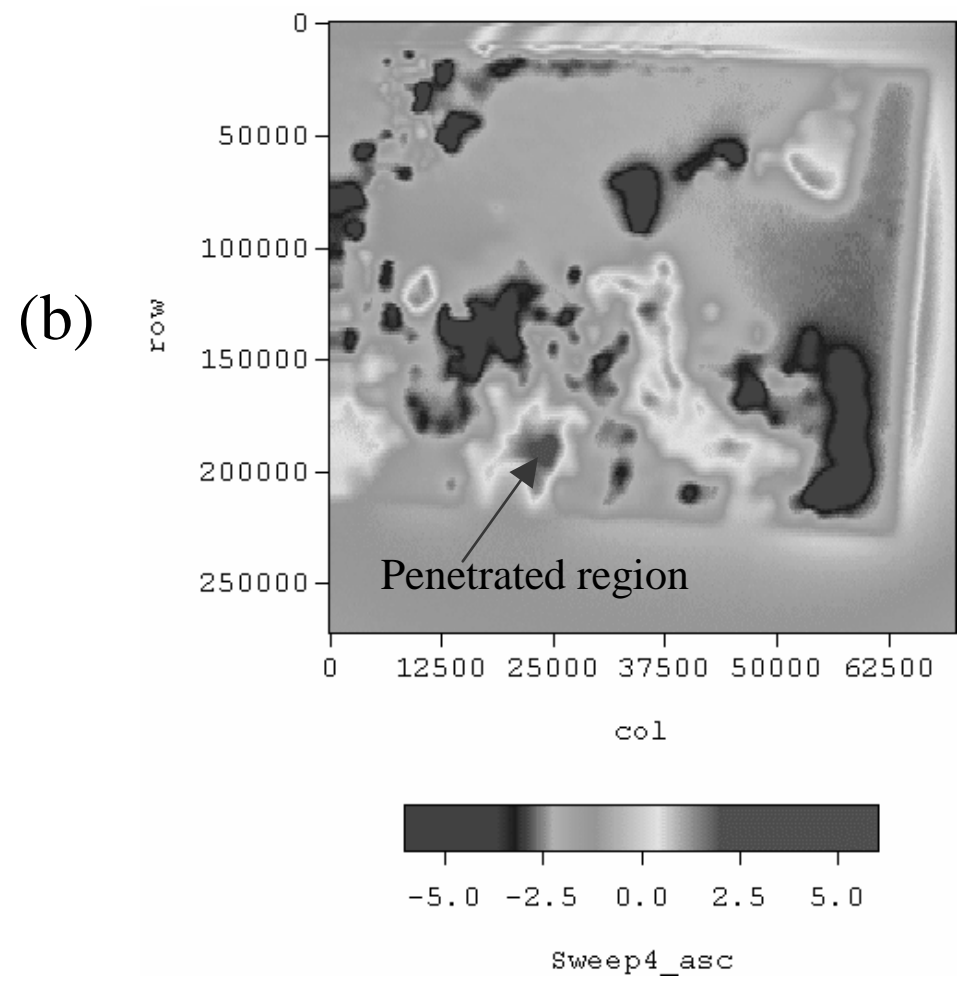

Figure 14. SSM images of a large region of sample, as the external magnetic field is increased at $\mathrm{T}=2 \mathrm{~K}$ for (a) $\mathrm{I}_{\text {coil }}=0.190$ $\mathrm{mA}$, and (b) $\mathrm{I}_{\text {coil }}=0.210 \mathrm{~mA}$.

This striking result is confirmed by scanning SQUID microscopy images. These successive following images, taken at $\mathrm{T}=4.2 \mathrm{~K}$, demonstrate that flux enters into the array in sort of catastrophic events. As the external flux is increased, nothing happens for the first steps. However, as can be seen in Fig. 14, all of a sudden flux 
streams into the array, following a catastrophic event.

We do not observe any dependency of the steps on the cooling/warming rate. Therefore, the appearance of the steps does not seem to be a phenomenon related to some kind of delay response of the sample. Moreover, we consider that the magnetic flux enters the sample when the critical current (the only one temperature dependent parameter) allows to have a matching between the geometry of the loops (through the parameter $\left.\beta_{L}\right)$ and an integer number of fluxons from the external available magnetic flux.

Further study should be necessary to undoubtedly confirm if the observed steps here described can be considered as flux avalanches related to a self-criticality mechanism. In particular, experiments for a fixed temperature should be necessary to confirm our findings. If so, it is obvious the enormous advantage to study these interesting phenomena in highly controllable devices such JJA instead of HTS single crystals in temperatures of the order of some $\mathrm{mK}$.

\section{Acknowledgments}

We thank M. G. Forrester, A. W. Smith and C. B. Whan for their technical help in the experiments. We also thank S. Sergeenkov, E. Granato, A. Sánchez and D. X. Chen for useful discussions.

We gratefully acknowledge financial support from U. S. Air Force Office of Scientific Research, through grant no. F496209810072 and from the National Science Foundation through grant no. 9510464.

F.M.A.M. also gratefully acknowledges financial support from Brazilian Agency FAPESP, under grants 96/7704-6 and 98/12809-7. W.M.J gratefully acknowledges financial support from Brazilian Agency FAPESP, under grants and 98/12593-4.

\section{References}

[1] F. M. Araujo-Moreira, O. F. de Lima, and W. A. Ortiz, Physica C, 240-245, 3205 (1994).

[2] F. M. Araujo-Moreira O. F. de Lima, and W. A. Ortiz., J. Appl. Phys. 80, 6, 3390 (1996).

[3] F. M. Araujo-Moreira O. F. de Lima, and W. A. Ortiz, Physica C 311, 98-106 (1999).

[4] W. A. C. Passos, P. N. Lisboa-Filho, R. Caparroz, C. C. de Faria, P. C. Venturini, F. M. Araujo-Moreira, S. Sergeenkov and W. A. Ortiz; Physica C 354, 189-196 (2000).

[5] R. S. Newrock, C. J. Lobb, U. Geigenmüller, and M. Octavio, Solid State Physics 54, 263 (2000).

[6] P. Martinoli and C. Leeman, J. Low temp. Physics 118, 699 (2000).

[7] R. B. Goldfarb M. Lelental, and C. A. Thomson, in Magnetic Susceptibility of Superconductors and Other Spin Systems, p. 49, edited by R. A. Hein, T. L. Francavilla and D. H. Liebenberg, Plenum Press, New York (1992).
[8] J. L. Jeanneret, G. A. Gavilano, A. Racine, Ch. Leemann, and P. Martinoli, Appl. Phys. Lett. 55, 2336 (1989).

[9] J. R. Kirtley, A. C. Mota, M. Sigrist, and T. M. Rice, J. Phys. Condens. Matter 10, L97 (1998).

[10] R. C. Black, A. Mathai, F. C. Wellstood, E. Dantsker, A. H. Miklich, D. T. Nemeth, J. J. Kingston, and J. Clarke; Appl. Phys. Lett. 62, 2128 (1993).

[11] A. Mathai, D. Song, Y. Gim, and F. C. Wellstood, Appl. Phys. Lett. 61, 598 (1992).

[12] F. M. Araújo-Moreira, P. Barbara, A. B. Cawthorne, and C. J. Lobb, Phys. Rev. Lett. 78, 4625 (1997).

[13] P. Barbara, F. M. Araujo-Moreira, A. B. Cawthorne, and C. J. Lobb, Phys. Rev. B 60, 7489 (1999).

[14] T. Wolf and A. Majhofer, Phys. Rev. B 47, 5383 (1993).

[15] W. Braunisch, N. Knauf, S. Neuhausen, A. Grutz, A. Koch, B. Roden, D. Khomskii, and D. Wohlleben, Phys. Rev. Lett. 68, 1908 (1992); W. Braunisch, N. Knauf, G. Bauer, A. Koch, A. Becker, B. Freitag, A. Grutz, V. Kataev, S. Neuhausen, B. Roden, D. Khomskii, and D. Wohlleben, Phys. Rev. B 48, 4030 (1993).

[16] L. N. Bulaevskii, V. V. Kuzii, and A. A. Sobyanim, JETP Lett. 25, 290 (1977); F. V. Kusmartsev, Phys. Rev. Lett. 69, 2268 (1992); H. Kauamura and M. S. Li, Phys. Rev. B 54, 619 (1996); D. Dominguez, E. A. Jagla, and C. A. Balseiro, Phys. Rev. Lett. 72, 2773 (1994).

[17] W. A. C. Passos, P. N. Lisboa-Filho, and W. A. Ortiz; Physica C 341-348, 2723 (2000).

[18] R. Lucht, H. v. Löhneysen, H. Claus, M. Kläser, and G. Müller-Vogt, Phys. Rev. B 52, 9724 (1995); S. Riedling et al., Phys. Rev. B 49, 13283 (1994).

[19] G. S. Okram, D. T. Adroja, B. D. Padalia, O. Prakash, and P. A. J. de Groot, J. Phys. C 9, L525 (1997).

[20] P. Kostic, B. Veal, A.P. Paulikas, U. Welp, V.R. Todt, C. Gu, U. Geiser, J.M. Williams, K.D. Carlson, R.A. Klemm, Phys. Rev. B 53, 791 (1996).

[21] I. Takeuchi, S. N. Mao, X. X. Xi, K. Petersen, C. J. Lobb, and T. Venkatesan, Appl. Phys. Lett. 67, 2872 (1995).

[22] I. Takeuchi, C. J. Lobb, Z. Trajanovich, P. A. Warburton, and T. Venkatesan; Appl. Phys. Lett. 68, 1564 (1996).

[23] T. Hashimoto, M. Sagoi, Y. Mizutani, J. Yoshida, and K. Mizushima; Appl. Phys. Lett. 60, 1756 (1992).

[24] C. P. Bean, Phys. Rev. Lett. 8, 250 (1962)

[25] C. P. Bean, Rev. Mod. Phys. 36, 31 (1964).

[26] H. London, Phys. Lett. 6, 162 (1963).

[27] H. Dersch and G. Blatter, Phys. Rev. B 38, 11391 (1988).

[28] Y. B. Kim, C. F. Hempstead, and A. R. Strnad, Phys. Rev. 129, 528 (1963). 
[29] W. A. Fietz, M. R. Beasley, J. Silcox, and W. W. Webb, Phys. Rev. 136, A335 (1964).

[30] M. Xu, D. Shi, and R. F. Fox, Phys. Rev. B 42, 10773 (1990).

[31] D. X. Chen, and R. B. Goldfarb, J. Appl. Phys. 66, 2489 (1989).

[32] D. X. Chen, A. Sanchez, and J. S. Muñoz, J. Appl. Phys. 67, 3430 (1990).

[33] J. L. Chen and T. J. Yang, Physica C 224, 345 (1994).

[34] J. R. Clem, Physica C 153, 50 (1988).

[35] M. Tinkham and C. J. Lobb, in Solid State Physics, edited by H. Ehrenreich and D. Turnbull, Academic, vol. 42, p. 91, New York (1989).

[36] C. A. Durán, P. L. Gammel, R. E. Miller, and D. J. Bishop, Phys. Rev. B 52, 75 (1995).

[37] P. G. de Gennes, in Superconductivity of Metals and Alloys, Ed. by W. A. Benjamin, New York (1966).
[38] P. Bak, C. Tang, and K. Weisenfeld, Phys. Rev. Lett. 59, 381 (1987); P. Bak, C. Tang, and K. Weisenfeld, Phys. Rev. A 38, 364 (1988).

[39] S. Field, J. Witt, F. Nori, and X. Ling, Phys. Rev. Lett. 74, 1206 (1995).

[40] C. M. Argenter, Phys. Rev. E 58, 1438 (1998).

[41] K. Behnia, C. Capan, D. Mailly, and B. Etienne, Phys. Rev. B, 61, 3815 (2000).

[42] R. Surdeanu, R. J. Wijngaarden, E. Visser, J. M. Huijbregtse, J. H. Rector, B. Dam, and R. Griessen, Phys. Rev. Lett. 83, 2054 (1999).

[43] R. Surdeanu, R. J. Wijngaarden, B. Dam, J. H. Rector, R. Griessen, C. Rossel, Z. F. Ren, and J. H. Wang, Phys. Rev. B 58, 12467 (1998).

[44] S. S. James, S. B. Field, S. Seigel, and H. Shtrikman, Physica C 332, 445 (2000). 